Kono. T

\title{
Cardiac rupture following catheter ablation procedure
}

\author{
Short Title: Cardiac rupture
}

Tetsuya Kono, MD, Hiroto Kitahara, MD, Masayuki Sakaguchi, MD, and Jun Amano, MD

Department of Cardiovascular Surgery, Shinshu University School of Medicine, Matsumoto, Japan

Key words: cardiac rupture, catheter ablation

Address reprint requests to: Tetsuya Kono, MD

Department of Cardiovascular Surgery, Shinshu University School of Medicine, Asahi 3-1-1, Matsumoto 390-8621, Japan

Phone: +81-263-37-2657

Fax: $\quad+81-263-37-2721$

E-Mail: tetsuya@hsp.md.shinshu-u.ac.jp 
Kono. T

\section{Abstract}

We described a patient who developed cardiac tamponade due to left ventricular rupture after a catheter ablation procedure. Rapid surgical exploration and use of cardiopulmonary bypass facilitated safe repair of the injury site and survival of the patient without complication. 
Kono. T

Cardiac rupture is a disastrous complication that leads to sudden death. Left ventricular rupture, often caused by myocardial infarction or chest trauma, brings high mortality and is a common finding at autopsy following myocardial infarction or blunt chest injury [1][2]. Left ventricular rupture as a complication of catheter ablation procedure is extremely rare. We report a case of a woman who survived rupture of the left ventricle following a catheter ablation procedure. Rapid surgical exploration after signs of cardiac tamponade and use of cardiopulmonary bypass (CPB) facilitated safe repair of the injury site and survival of the patient without complication.

A 61-year-old female presented with a history of recurrent supraventricular tachycardia. Since tachycardia episodes were poorly controlled by pharmacological treatment, she underwent a catheter ablation procedure. Three 5 Fr electrode catheters were positioned at the high right atrium, His-bundle area, and right ventricular apex through the left femoral vein. After these attempts, a 6 Fr electrode catheter was introduced percutaneously through the right femoral vein and advanced into the coronary sinus. Sustained orthodromic reciprocating tachycardia was induced with programmed stimulation, and multiple endocavitary electrograms were simultaneously obtained. The sequence of retrograde atrial activation during tachycardia demonstrated the earliest atrial electrogram in the distal coronary sinus, conforming to the left-lateral location of the accessory pathway (AP), and was therefore diagnosed as concealed Wolff-Parkinson-White syndrome. Heparin was administered intravenously and a 6 Fr mapping and ablation catheter was inserted through the right femoral artery to identify the ablation target site by mapping the ventricular aspect of the mitral annulus. During ablation maneuvers, the patients experienced syncope, bradycardia, and hypotension [80 mmHg, systolic]. Fluoroscopy showed reduced excursions of the cardiac silhouette. Protamine was administered, and the procedure was interrupted to perform transthoracic echocardiography (TTE) under suspicion of cardiac tamponade. While TTE showed $8 \mathrm{~mm}$ echo-free space surrounding the heart, the ventricles were normal in shape and function. Furthermore, the patient was recovered by temporary RV pacing and low dose cathecholamine administration. Since she remained hemodynamically stable for half an hour in the catheterization laboratory, the patient was transferred to the intensive care unit and 
Kono. T

observed carefully. She had remained hemodynamically stable, thereafter, and the ventricles were normal in shape and there was no increase in the echo-free space demonstrated by TTE 3 hours, 6 hours, and 24 hours after catheter ablation, respectively. Thirty-six hours after catheter ablation, however, she suddenly developed circulatory collapse due to cardiac tamponade. Emergency surgery was proposed and she was transferred to the operating room quickly.

Median sternotomy was performed. A large volume of clot was found around the right atrium. After removal of clots, bright red blood was noted coming from the left side of the pericardium, although there were no tears of the right atrium or right ventricle found. Thus, we diagnosed left-sided cardiac rupture. CPB with ascending aorta cannulation and venous cannulation of the right atrium was established quickly. After cardioplegic arrest, the left ventricle was inspected. Left ventricular tear was found in the posterolateral wall of the left ventricle. This tear was $3 \mathrm{~cm}$ long and lying between the posterolateral branches of the left circumflex artery. The tear was repaired with Teflon-buttressed sutures (Fig. 1). Then CPB was discontinued without difficulty and with excellent hemodynamic status. Postoperatively, the patient did not experience any complication and was discharged from the hospital on the 14th postoperative day.

\section{Comment}

Catheter ablation has developed into a new non-pharmacological therapy for definitive treatment of patients with cardiac arrhythmias. Catheter ablation of the accessory pathway with radiofrequency energy has been performed with a high success rate and low incidence of complications [3][4]. In radiofrequency catheter ablation, radiofrequency energy is delivered from an external generator and destroys tissue by controlled heat production [5] and complications such as atrioventricular block, right atrial thrombus, pericarditis, coronary spasm, cardiac tamponade have been reported in the literature [6][7]. Hindricks reported that in patients with left-sided atrioventricular AP, placement of electrode catheter into the coronary sinus may damage the small branch of the coronary vein and may result in cardiac tamponade [8]. Cardiac tamponade due to left venticular 
Kono. T

rupture following catheter ablation procedure, however, is extremely rare, and, to our knowledge, has not been previously reported in the literature. In our case, cardiac rupture was considered to begin from a small leak through friable myocardium, causing oozing, followed by gradual spread and perforation into the pericardial cavity, causing "blow out” rupture. Pericardial effusion due to the oozing from the ablation site may have resulted in hypotension in the catheterization laboratory. Thirty-six hours after catheter ablation, she developed circulatory collapse due to "blow out" cardiac rupture.

The success rate in managing cardiac injuries depends on early recognition of its severity through careful observations and diagnosis. The presence of signs of cardiac tamponade or continued bleeding following catheter ablation procedure should be considered indications for early surgical exploration. Though atrial tears have been managed without cardiopulmonary bypass, instituting CPB during surgery is important in dealing with this life-threatening situation. Because CPB stabilizes the hemodynamic state if active bleeding is present after opening the pericardium and facilitates secure repair under an empty relaxed ventricular condition [9]. In our case, after removal of clots, bright red blood coming from the left side of the pericardium was noted while there was no right atrial or right ventricular injury. Therefore, we considered that the injury was left-sided cardiac rupture, and quickly established CPB. The use of CPB facilitated safer and quicker repair of the injury site, and avoided circulatory deterioration during surgical repair.

In conclusion, we describe a woman who survived rupture of the left ventricle following catheter ablation. Rapid surgical exploration after signs of cardiac tamponade and the use of CPB facilitated safe repair and survival of the patient without complications. 


\section{References}

1. Bashour T, Kabbani SS, Ellertson DG, Crew J, Hanna ES. Surgical salvage of heart rupture: report of two cases and reiew of the literature. Ann Thorac Surg 1983; 36: 209-213

2. Kato K, Kushimoto S, Mashiko K, et al. Blunt traumatic rupture of the heart. A experience in Tokyo. J Trauma 1994;36: 859-864

3. Scheinman, MM. Patterns of catheter ablation practice in the United States: Result of the1992 NASPE survey. PACE 1994; 17: 873-5

4. Hindricks G, on behalf of the Multicentre European Radiofrequency Survey [MERFS] Investigators of the Working Group on Arrhythmias of the European Society of Cardiology: The Multicentre European Radiofrequency Survey [MERFS]: Complications of radiofrequency catheter ablation of arrhythmias. Eur Heart J 1993; 14: 1644

5. Douglas P. Zipes. Management of cardiac arrhythmia: pharmacological, electrical, and surgical techniques. In: Eugene Baunwald ed. Heart disease $5^{\text {th }}$ ed. Philadelphia: Saunders, 1997: 593-639. 6. Jackman WM, Wang X, Friday KJ, et al. Catheter ablation of accessory pathways (Wolff-Parkinson-White syndrome) by radiofrequency current. N Engl J Med 1991; 324: 1605-1611 7. Calkins H, Langberg JJ, Sousa J, et al. Radiofrequency catheter ablation of accessory atrioventricular connections in 250 patients: abbreviated therapeutic approach to Wolff-Parkinson-White syndrome. Circulation 1992; 85: 1337-1346

8. Hindricks G. Incidence of procedure-related complications of radiofrequency catheter ablation: Are there significant differences between "low volume” and "high volume” centers (abstract) Circulation 1993; 88: I-296

9. Yaku H, Fermanis G, Horton DA, Guy D, Lvoff R. Successful repair of a ruptured postinfarct pseudoaneurysm of the left ventricle. Ann Thorac Surg 1995; 60: 1097-1098 
Kono. T

\section{Figure legends}

Fig 1. Operative Schema

The tear lying between posterolateral branches of the left circumflex artery was repaired with

Teflon-buttressed sutures. (PL indicates the posterolateral branch of the left circumflex artery.) 